\title{
Esophagectomy and splenectomy in a patient with osteopetrosis
}

\author{
Samantha K. Hendren, MD, ${ }^{a}$ Jessica Wang, MD, ${ }^{a}$ Joseph Gorman, MD, ${ }^{a}$ Thomas Peacock, MD, ${ }^{c}$ \\ Diane M. Hershock, MD, PhD, ${ }^{b}$ and Ernest F. Rosato, MD, ${ }^{a}$ Philadelphia, $\mathrm{Pa}$
}

$\mathrm{O}$ steopetrosis is a rare genetic disorder characterized by a failure of osteoclast-mediated bone resorption and by hematologic abnormalities. A patient with osteopetrosis presenting with a distal esophagus cancer and secondary hypersplenism was surgically treated.

\section{Clinical Summary}

The patient is a 57-year-old man with osteopetrosis since childhood, complicated by multiple orthopedic fractures (Figure 1), osteomyelitis, splenomegaly, thrombocytopenia, and chronic anemia requiring routine blood transfusions. Several months before diagnosis, he was noted to have an increasing transfusion requirement. The hemoglobin fell to $2.6 \mathrm{~g} / \mathrm{dL}$, and upper endoscopy demonstrated bleeding from an adenocarcinoma at the gastroesophageal junction (Figure 2).

An exploratory procedure was performed through a left thoracoabdominal incision. The diaphragm was divided circumferentially. A splenectomy was performed with a subsequent intraoperative platelet transfusion. The esophagus was divided at the level of the inferior pulmonary vein and removed en bloc with the proximal stomach and associated lymphoid tissue. The anastomosis was created by using a combined hand-sewn and stapled technique. Pathologic examination revealed a poorly differentiated adenocarcinoma, measuring $3.3 \mathrm{~cm}$ and invading the submucosa, with lymphovascular invasion and metastasis in 1 of 14 lymph nodes. The enlarged spleen demonstrated extramedullary hematopoiesis.

The patient tolerated the procedure well. His postoperative course was complicated by a pulmonary embolism, treated with a vena cava filter. He was discharged on postoperative day 10 eating a regular diet, with a dramatically reduced requirement for blood and platelet transfusion. The total utilization of blood products was 13 units of packed red blood cells, 13 units of platelets, and 2 units of fresh frozen plasma.

After discharge from the hospital, the patient's quality of life was substantially improved owing to resolution of symptoms, as well as a durable elimination of his transfusion requirement. Ap-

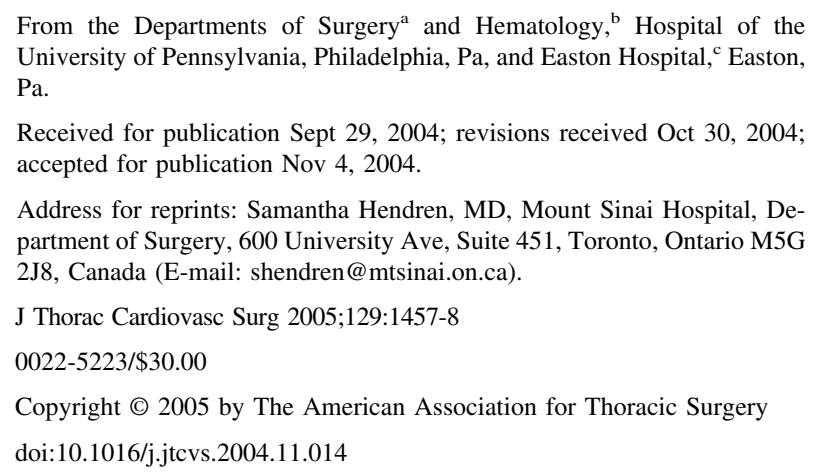

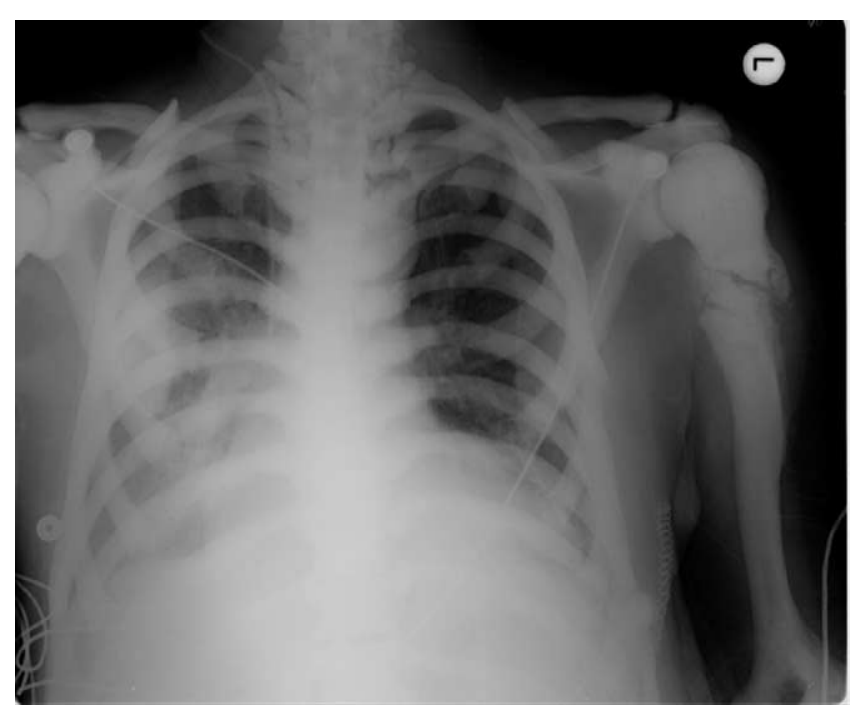

Figure 1. Preoperative chest $x$-ray film reveals the dense skeleton of osteopetrosis and a chronic left humeral fracture.

proximately 18 months after his operation, weight loss and functional decline ensued due to liver metastases; 22 months after his operation, he died of progressive metastatic disease.

\section{Comment}

Although all patients with osteopetrosis have dense, fragile bones and obliteration of the marrow space, other manifestations of the disease vary. Osteopetrosis has traditionally been separated into 2 forms: infantile "malignant" and adult "benign."1 Clinically, the infantile form manifests with rickets-like bony changes, hepatosplenomegaly, progressive pancytopenia, cranial neuropathies, and susceptibility to infection. ${ }^{2}$ This disease is lethal in infancy or early childhood, except in some children treated with human leukocyte antigen-matched bone marrow transplantation.

The adult, benign form of osteopetrosis may be asymptomatic, or pathologic fractures and osteomyelitis may occur. Severe hematologic disturbances and hepatosplenomegaly are usually absent. ${ }^{3}$ The patient described here does not fit easily into the infantile or adult form of osteopetrosis and probably had an attenuated form of the infantile disease or an intermediate type of osteopetrosis.

Splenectomy has been used as a palliative procedure for patients with infantile osteopetrosis and hypersplenism since the 1960s. ${ }^{4}$ The primary concern with a splenectomy is the removal of a major site of extramedullary hematopoiesis in patients with little functioning bone marrow. To predict which patients will benefit from a splenectomy, one may use hematologic and radionuclide (bone marrow scintigraphy, red blood cell survival) studies to 


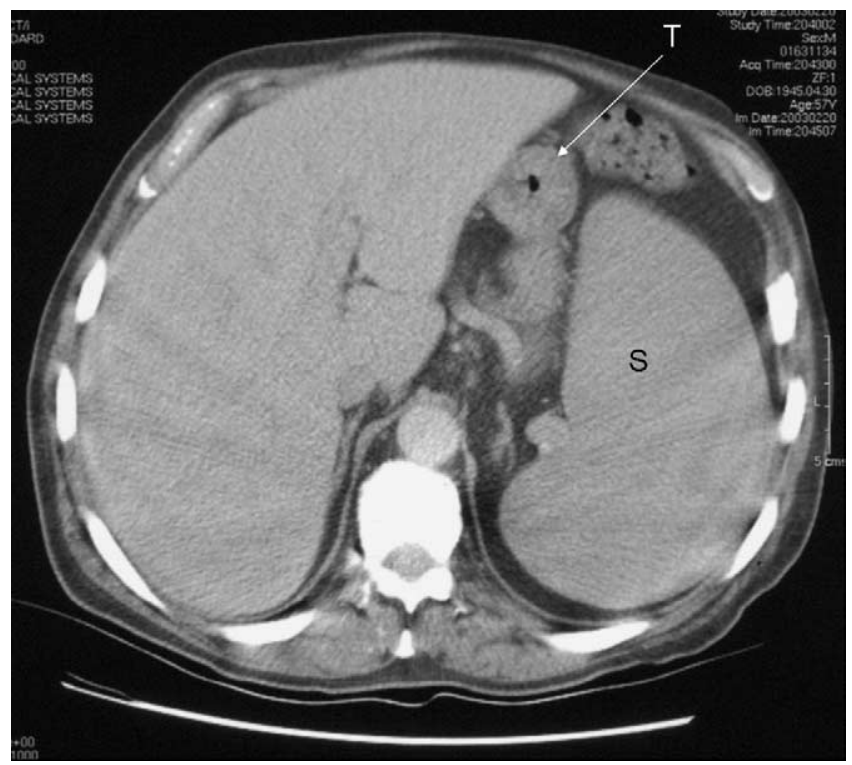

Figure 2. Abdominal computed tomographic scan showing esophageal lesion and splenomegaly. $S$, Spleen; $T$, tumor.

determine the relative contributions of decreased red cell production and increased destruction to a patient's anemia and thrombocytopenia. ${ }^{2,4,5}$

Although this patient's comorbidities substantially increased his risk for perioperative complications, we felt that surgery was the best treatment option because we believed that the patient's severe bleeding and hypersplenism could be most effectively addressed with surgery. We also believe that surgery provides the best chance for cure in locally advanced esophageal cancer, while avoiding potential bone marrow toxicity from nonoperative cancer treatments. In this case, we decided on a left thoracoabdominal approach because we believed that the patient's bleeding diathesis placed him at risk for excessive bleeding with blunt esophageal dissection. In addition, the thoracoabdominal approach provides superior exposure to the enlarged spleen compared with an anterior abdominal incision.

Additional considerations when operating on a patient with osteopetrosis include prevention of iatrogenic fractures and dental injuries, to which these patients are extremely susceptible, and perioperative correction of anemia and thrombocytopenia. Collaboration with a hematologist is essential. Adjuvant therapy was not prescribed because it was thought that the risk of hematologic suppression in this patient was too great to justify the potential benefit.

In summary, this is a report of esophagectomy and splenectomy performed for esophageal adenocarcinoma and secondary hypersplenism in a patient with osteopetrosis. With proper preparation and precautions, this high-risk patient underwent a major operation with favorable early outcome. Suggestions for perioperative management of the patient with osteopetrosis are made, and the palliative potential of splenectomy in osteopetrosis is highlighted.

\section{References}

1. Marks SC. Congenital osteopetrotic mutations as probes of the origin, structure, and function of osteoclasts. Clin Orthop. 1984;189:239-63.

2. Wilson CJ, Vellodi A. Autosomal recessive osteopetrosis: diagnosis, management, and outcome. Arch Dis Child. 2000;83:449-52.

3. Shapiro F. Osteopetrosis: current clinical considerations. Clin Orthop. 1993;294:34-44.

4. Besselman DM. Splenectomy in the management of the anemia and thrombocytopenia of osteopetrosis (marble bone disease). J Pediatr. 1966;69:455-7.

5. Moe PJ, Skjaeveland A. Therapeutic studies in osteopetrosis. Report of 4 cases. Acta Paediatr Scand. 1969;58:593-600. 